\title{
A measurement-based approach for power system instability early warning
}

\author{
Feifei Bai ${ }^{1}$, Yong Liư ${ }^{2}$ Yilu Liư ${ }^{2,3}$, Kai Sun ${ }^{2}$, Navin Bhatt ${ }^{4}$, Alberto Del Rosso ${ }^{4}$, Evangelos Farantatos ${ }^{4}$ \\ and Xiaoru Wang ${ }^{1 *}$
}

\begin{abstract}
Early warning of impending instability in a power system under disturbance conditions is important for preventing of system collapse. A measurement-based approach is proposed to assess the potential power system transient instability problem under cascading outages. Where a measurement-based index is obtained as the estimation accuracy of a linear autoregressive exogenous (ARX) model to estimate the dynamic response of the power system and indicate the system stability to some extent after a disturbance. The proposed approach was verified using a set of marginally stable cases in a 179-bus WECC equivalent power system. Then the instability early warning threshold for this system is obtained as 0.44.
\end{abstract}

Keywords: Autoregressive exogenous model, Accuracy index, Dynamic response estimation, Instability early warning, Measurement-based

\section{Introduction}

With the interconnections of power system increasing, the instability assessment is becoming more and more important for safe operation of power systems. The power system stability refers to the continuance of intact operation following a disturbance, it depends on the operation and the nature of the physical disturbance [1, 2]. The 2003 blackout occurred in N.E. North America is partly because of the lack of supportive applications when the system is close to instability [3]. If power system is close to the stability limit, actions must be taken by system operators to identify critical states. Therefore, it is very important to have an index for the critical situation awareness. A series of disturbances can increasingly stress the system, degradation of its stability margin may finally lead to loss of stability. Therefore, the analysis of system dynamic response during or after the disturbance is very important to indicate the potential instability of the power system [4].

The conventional methods of power system analysis are based on numerical solution of system differential algebraic equations. These numerical methods need more detailed representation of the power system but they are not suited for online application of stability

\footnotetext{
* Correspondence: xrwang@home.swjtu.edu.cn

'Southwest Jiaotong University, Chengdu, Sichuan 610031, China

Full list of author information is available at the end of the article
}

assessment and control. There are three reasons: 1) The power system dynamic model could not include all the details of the power system; 2) The topology of the power system changes all the time; 3) The long computation time of the numerical solution. With a large number of synchrophasors being deployed, it is possible to construct power system model based purely on real-time synchrophasor measurements, which also can make online power system instability awareness feasible. Studies have been done with the measurement data for the system instability assessment [5-11]. Some focus on using real-time phasor measurements with pre-existing knowledge obtained from computer simulation results or historical events to enable real-time assessment under disturbance conditions $[8,9]$. Some use selected realtime measurement locations to directly compute energy functions for the potential of instability [10, 11]. An adaptive power system equivalent method for real-time estimation of stability margin using phase-plane trajectories was proposed in [7]. This paper proposes a new method for instability early warning only using continuous high-sampling-rate measurements. In order to create the system collapse case, the idea in [7] using a series of disturbances to stress the system was used in this paper. Then an index was proposed to assess the potential instability of the system. 
The rest of the paper is organized as follows. Section II is the introduction of the measurement-based system dynamic response estimation model and the model accuracy index. Section III is to implement the proposed model accuracy index to evaluate the system instability. Section IV is the case study of the proposed method. Conclusion and future work are provided in Section V.

\section{Methodology of the proposed approach Dynamic response estimation}

A concept of dynamic response estimation for power system was proposed in [12]. It is to estimate the dynamic response of the power system during or after disturbances. The basic idea of the dynamic response estimation is to identify the real-time dynamic model or the transfer function of the power system and use the obtained model to estimate the dynamic response of the power system.

\section{The ARX model structure}

Two categories of measurement-based models can be used for system identification: state-space model [1317], and transfer function model [18-21]. The statespace representation is concerned not only with input and output properties of the system but also with its complete internal behavior [22]. In contrast, the transfer function representation is concerned with and specifies only the input/output behavior. Hence, the transfer function model identification can be an alternative to overcome the drawback of high computation burden of state-space methods. The linear Auto Regressive Exogenous model (ARX) provides a much simpler identification model of multi-variable system than the statespace model or other models. The general expression of the transfer function model structure [23] is:

$$
A(z) y(t)=\frac{B(z)}{F(z)} u(t)+\frac{C(z)}{D(z)} e(t)
$$

where

$A(z)=1+a_{1} z^{-1}+\ldots+a_{n_{a}} z^{-n_{a}}, B(z)=b_{1} z^{-1}+\ldots+b_{n_{b}} z^{-n_{b}}$

$C(z)=1+c_{1} z^{-1}+\ldots+c_{n_{c}} z^{-n_{c}}, D(z)=1+d_{1} z^{-1}+\ldots+d_{n_{d}} z^{-n_{d}}$

$F(z)=1+f_{1} z^{-1}+\ldots+f_{n_{f}} z^{-n_{f}}$

where $t$ is the time index, and $e(t)$ is a white noise, $z^{-1}$ is a backward shift operator and $z^{-1} y(t)=y(t-1) . n_{a}, n_{b}$, $n_{c}, n_{d}, n_{f}$ are the orders of the signal $y(t), u(t)$, and $e(t)$, respectively. If $C(q)=1, D(q)=1$ and $F(q)=1$, then (1) becomes to be the AutoRegressive model with eXogenous inputs (ARX) model. The mathematical structure expression of the ARX model is also can be described by the equation:

$$
\begin{aligned}
& y(t)+a_{1} y(t-1)+\cdots+a_{n_{a}} y\left(t-n_{a}\right) \\
& =b_{0} u(t)+b_{1} u(t-1)+\cdots+b_{n_{b}} u\left(t-n_{b}\right)+e(t)
\end{aligned}
$$

With the SISO ARX model structure (1), the multiinput single-output (MISO) ARX model structure can be derived:

$$
\begin{aligned}
& y(t)+a_{1} y(t-1)+\cdots+a_{n_{a}} y\left(t-n_{a}\right) \\
& =\sum_{j=1}^{M}\left(b_{j 0} u_{j}(t)+b_{j 1} u_{j}(t-1)+\right. \\
& \left.\cdots+b_{j n_{b j}} u_{j}\left(t-n_{b j}\right)\right)+e(t)
\end{aligned}
$$

For the simplification, (3) can be further expressed in the vector form as:

$$
\mathbf{a y}(t)=\sum_{j=1}^{M} \mathbf{b}_{j} \mathbf{u}_{j}(t)+\mathrm{e}(t)
$$

where

$\mathbf{a}=\left[1, a_{1}, \cdots, a_{n_{a}}\right], \mathbf{y}(t)=\left[\mathrm{y}(t), \mathrm{y}(t-1), \cdots, \mathrm{y}\left(t-n_{a}\right)\right]$

$\mathbf{b}_{j}=\left[b_{j 0}, b_{j 1}, \cdots, b_{j n_{a j}}\right], \mathbf{u}_{j}(t)=\left[u_{j}(t), \cdots, u_{j}\left(t-n_{b j}\right)\right]$

Because of the linear structure of the ARX model, the model parameters of a multi-input ARX model can be estimated by a linear Least Square (LS) approach. The objective function is

$$
\operatorname{Min} J\left(V_{L S}\right)=\sum_{\mathrm{k}=n_{\mathrm{s}}+1}^{N}(\hat{y}(k)-y(k))^{2}
$$

where $N$ is the total data points, $\hat{y}(k)$ and $y(k)$ are the actual response and estimated response, respectively.

\section{Model accuracy index}

To evaluate the identified ARX model, a fitness criterion can be performed [24]:

$$
F=\left(1-\frac{\sqrt{(\hat{Y}-Y)^{2}}}{\sqrt{(\hat{Y}-\bar{Y})^{2}}}\right) \times 100
$$

where $Y, \hat{Y}, \bar{Y}$ are the estimated response by the ARX model, measured response by PMU/FDR, and the mean value of the measured response, respectively. This index is the accuracy of the model estimation in describing system dynamic characteristics. A fitness of 100 means a perfect fit between the estimated response and the actual response, while a fitness of zero means the estimated response $\mathrm{Y}$ is no better than the mean value of measured response $\bar{Y}$.

For easier interpretation, a normalization process that converts the accuracy index from $(-\infty, 100]$ to $(0,1]$ can be performed as followed: 


$$
A=e^{(F / 100)-1}
$$

where $F$ is the fitness index defined in previous study and $A$ is the normalized accuracy index. The accuracy index of instability assessment threshold can be determined as:

$$
T=A_{\max }
$$

where $A_{\max }$ is the maximum one among all the calculated accuracy index in marginally stable cases. $T$ is the maximum value of the accuracy index of the last disturbance before instability. The accuracy represents the difference between the actual response at the output location and the response estimated by the ARX model.

\section{Method for instability awareness}

\section{Capability of accuracy index for instability warning}

The application of the linear ARX-structured modeling method is under the assumption that the power system to be modeled is relatively linear around certain operation points. Although the power system is nonlinearin nature, the operating point of the large-scale power grid does not change dramatically generally. The power system shows linear characteristics most of the time and thus ARX-structured method may have merit in bulk power grid modeling [24]. However, when a series of disturbances are increasingly stressing the system, the system will become less and less stable so that the trained ARX model will tend to be less accurate to estimate the dynamic response under the changed topology, which will be reflected as the accuracy index becoming lower. The threshold for the instability early warning was obtained when the system under the marginally stable situation after cascading outage. Marginally stable means the system is pushed to the edge of the stability, no matter how small a disturbance is added to this system, the system will collapse. The threshold is the estimation accuracy index of the marginally stable case.

\section{Guideline for instability early warning}

While a series of disturbances are increasingly stressing the system, degradation of its stability margin may finally

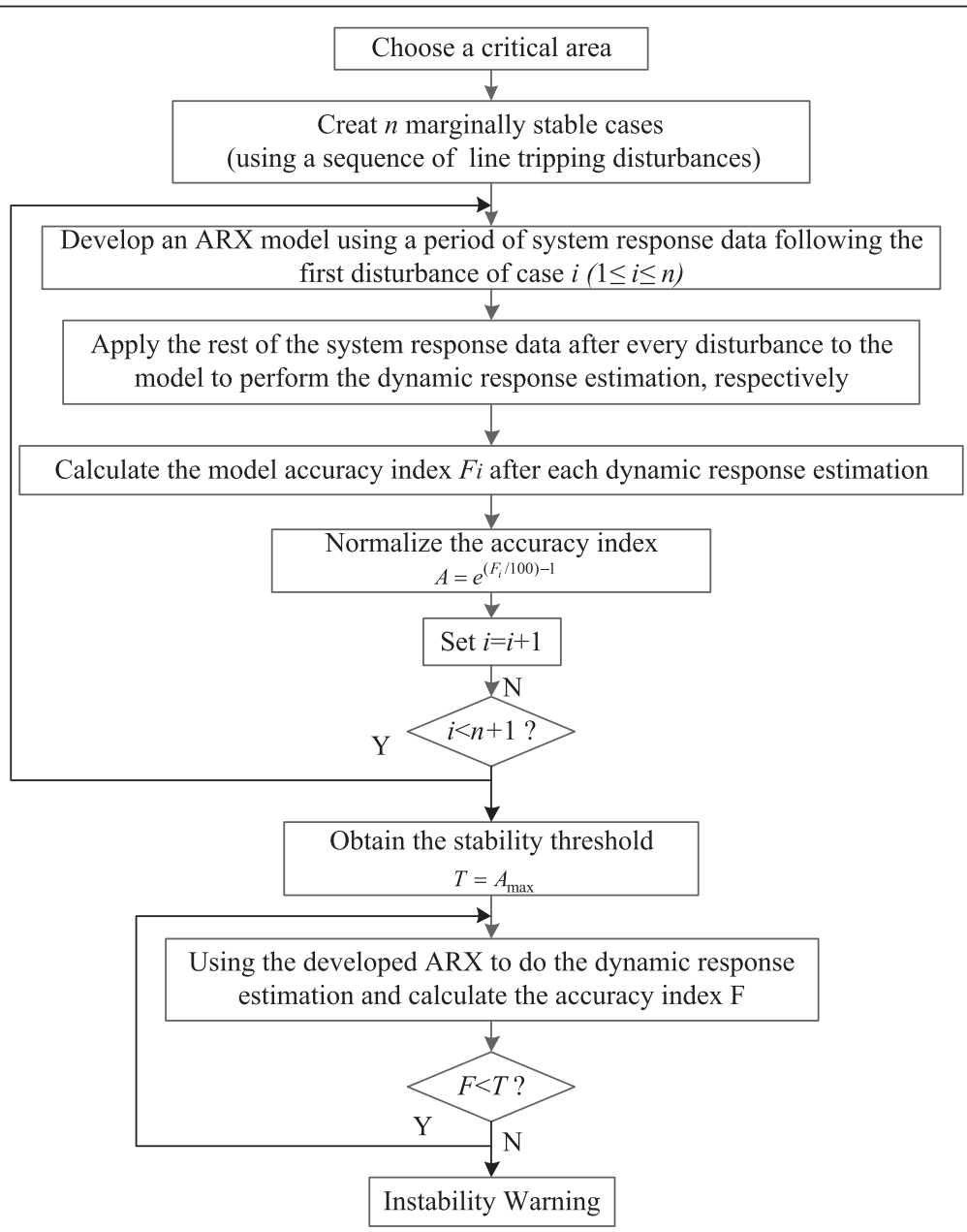

Fig. 1 Flowchart for system instability assessment 
lead to the system collapse. Continuous measurements on the monitored variables at a high sampling rate enable the ARX dynamic model to estimate the dynamic response in real time, and the model accuracy index obtained from the ARX dynamic model can indicate the decreasing of the stability margin. Therefore, a precisely defined threshold for instability early warning is necessary. Incidents of instability on a power system are not many, but they generally are accompanied by severe consequences. Therefore, the off-line dynamic simulation is the most convenient way to derive the threshold of the model accuracy index.

The method developed to assess potential system instability is described in Fig. 1. The basic steps of this flowchart are:

(1)For a specific critical area, create $N$ marginally stable simulation cases using a sequence of line faults.

(2)For each simulation case, find the interface of the critical area and choose the input and out channel in each side of the interface, respectively. Then develop an ARX model using a period of system response data following the first disturbance.

(3) After obtaining such a model, the system responses following the rest of the disturbance sequence are applied to the model to perform the response estimation.

(4) Accuracy index values are obtained after disturbances.

(5) Repeat Step (2), (3) and (4) for $N$ marginally stable cases.

(6) The threshold could be determined as the largest accuracy index of the last disturbance before collapse in all the $N$ marginally stable cases.

(7) Using the developed model to estimate the system response, if the accuracy index is equal or smaller than the threshold, a warning signal indicating potential instability following the next disturbance will be generated.

\section{Case study for system instability early warning}

Section III illustrated the proposed accuracy index threshold derivation process. To validate the idea of using the model accuracy index to early warn potential instability, an equivalent 179-bus WECC model [25] is used as the test system, as shown in Fig. 2. This model is simplified but retains the main dynamics of the entire WECC system.

\section{Marginally stable case}

Marginally stable means the system is pushed to the edge of the stability, no matter how small a disturbance is added to this system, the system will collapse [7].

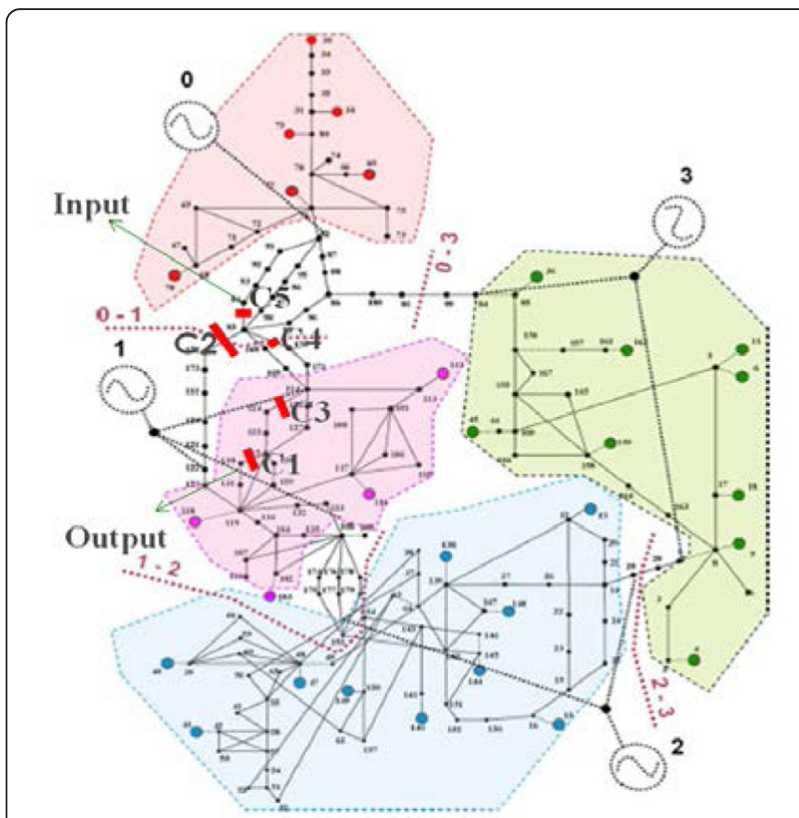

Fig. 2 WECC 179-bus model and disturbance locations

Using the same approach as in [7] to create the marginally stable cases, a sequence of three-phase line faults is used in this case to drive the interface between area " 0 " and " 1 " to its marginally stable point. As an example, a marginally stable case is created by five faults occurring at different locations $(\mathrm{C} 1, \mathrm{C} 2, \mathrm{C} 3, \mathrm{C} 4$ and $\mathrm{C} 5$ in Fig. 2). The first four clearing time of line faults was fixed (five cycles), and the last one was adjusted to make this case to be marginally stable, which means the system would collapse if the clearing time of the last fault increase by $0.001 \mathrm{~s}$. A lot a simulation cases were carried out using this method, only eight marginally instable cases can be created. Please note that each fault is cleared by opening the fault line. They increasingly weaken the interface but do not break the connection. This case can increasingly stress the operating condition (weakening the topology around that interface) by series of permanent faults.

The bus frequency, voltage magnitude and power angle simulation results in one marginally stable case are shown in Fig. 3. From Fig. 3, after five contingencies, the system has not caused a collapse yet. However, it has been pushed to its marginally collapse point, because if the clearing time of the last fault is increased by $0.001 \mathrm{~s}$, the system would lose transient stability right after the last fault (in Fig. 4). Therefore, the interface between area " 0 " and "1" has been pushed to its marginally stable point.

\section{Threshold for instability early warning}

A threshold is needed to alert the system operator in order to prevent the system being pushed to instability. 

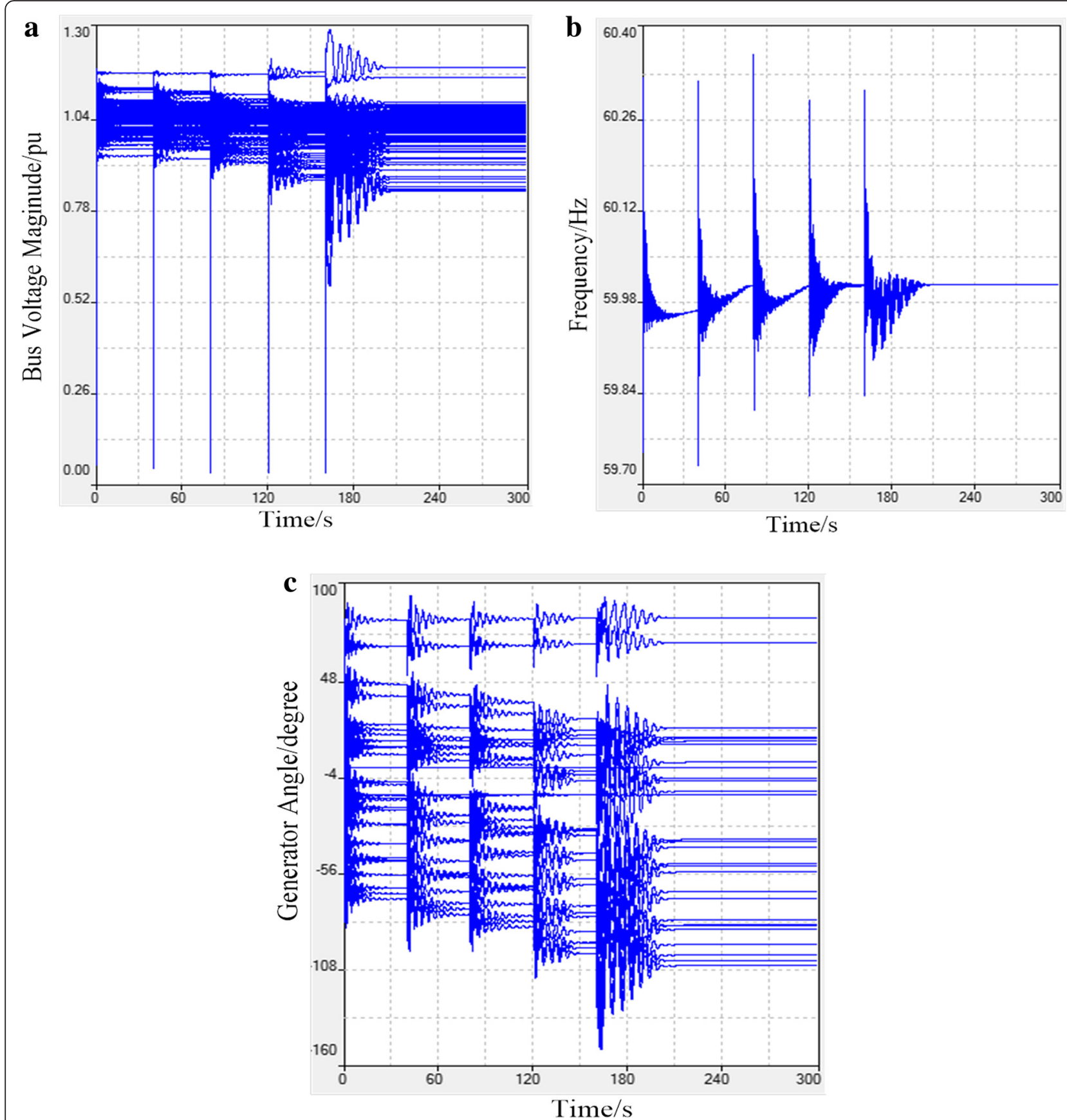

Fig. 3 Dynamics response of marginally stable case. a Voltage dynamic response after disturbance. b Frequency dynamic response after disturbance. c Power angle dynamic response after disturbance

The proposed threshold determination method is applied in this section by investigating the instability problem between areas " 0 " and other areas.

\section{Case study and results}

In this study, the inputs of the ARX model is located in area "0" and the output is located in area "1", which are shown in Fig. 2. The first disturbance is the three phase line fault occurred between area " 0 " and area "1" at location "C1" marked in Fig. 1, which is used to train the ARX model and obtain the first accuracy index of the model. Then four disturbances occur in every $40 \mathrm{~s}$ at different locations $(\mathrm{C} 2, \mathrm{C} 3, \mathrm{C} 4$ and $\mathrm{C} 5$ in Fig. 2) following the first disturbance. Frequency dynamic response after every disturbance is obtained and the accuracy index is calculated at the 

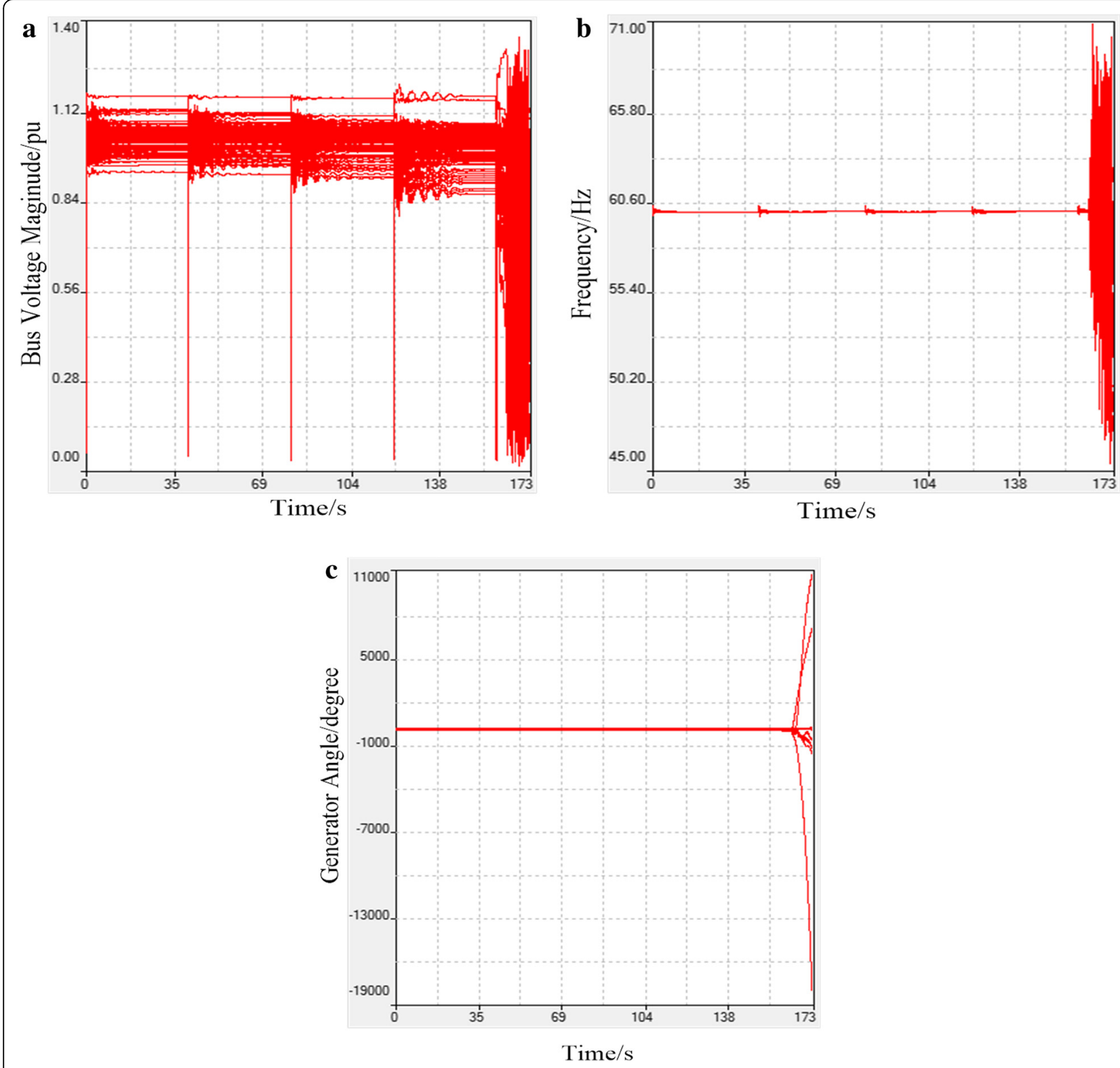

Fig. 4 Dynamic responses of 29 generator buses. a Voltage dynamic response after disturbance. b Frequency dynamic response after disturbance. c Power angle dynamic response after disturbance

same time. The estimation results and accuracy index calculation results are shown in Fig. 5.

As shown in Fig. 5, the system tends to be less and less stable as more line disconnection disturbances added into the system, which is accompanied by the accuracy index of the system dynamic response decreasing, which indicates that the model accuracy index can be used to indicate the system stability to some extent.

In order to obtain the threshold of the accuracy index for transient instability early warning, a lot of simulation case studies were carried out, here a set of eight scenarios was created to provide a comprehensive coverage of stability performance of the power system. The variation trends of accuracy indices following the sequences of disturbances in eight scenarios are shown in Fig. 6.

Most of the accuracy indices in all the cases decrease due to the sequences of disturbances in Fig. 6. The difference of the disturbance location may be the main factor for special case 4 and case 8 why did not keep decreasing all the time. For all the simulation cases, after the fifth disturbance, the system is pushed to be marginally stable and most of the accuracy index also reaches its lowest point at the same time. The threshold can be obtained by the majority situations. It should be noted that this is a preliminary investigation on threshold value determination for system transient instability early warning. More studies need to be done to 


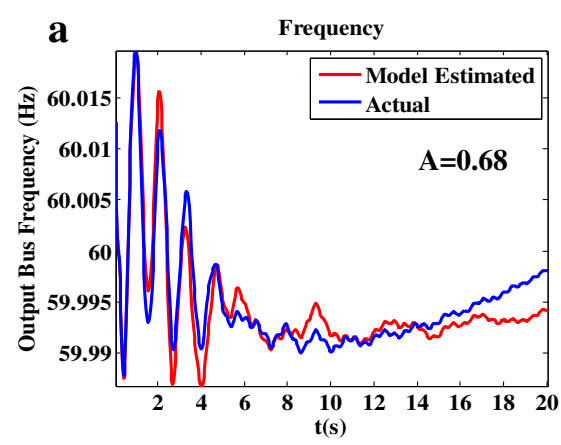

Contingency 1

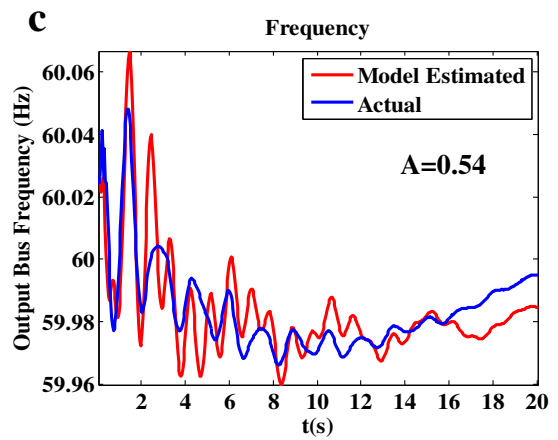

Contingency 3

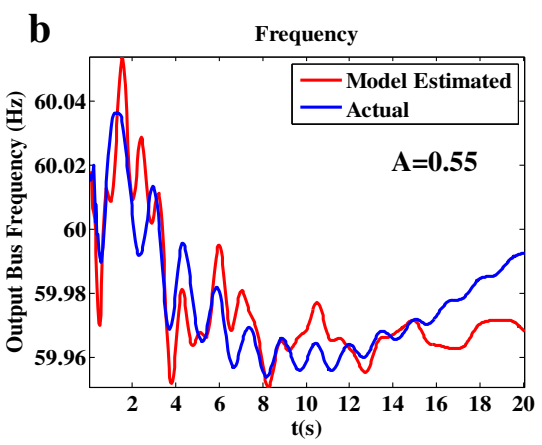

Contingency 2

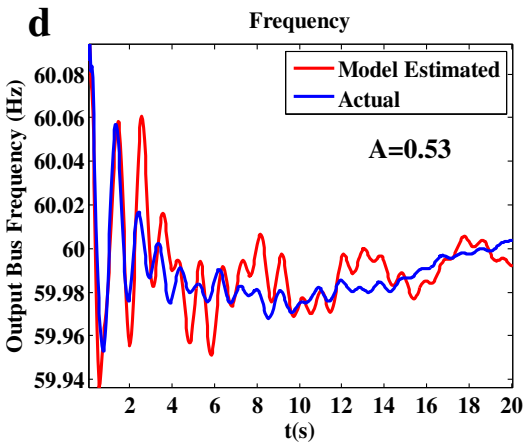

Contingency 4

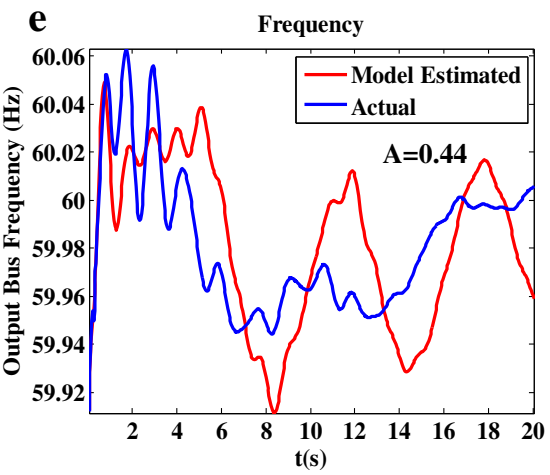

Contingency 5

Fig. 5 Dynamic response and accuracy index after contingency

obtain further insight into the relationship between accuracy indices and potential instability for future work. The study in this paper is only an example to show how to derive the threshold with the proposed method.

For step 6 of the method described above, the threshold for this particular application is suggested as 0.44, the largest accuracy index value before collapses, as shown in Fig. 6. It means when the accuracy index reached 0.44 , the system is almost stressed to its marginally instable point. For other cases, events with accuracy indices less than 0.44 are not necessarily instable, however, severe enough to alert for potential instability. This particular threshold selection method here only gives a "safe zone" of system operation, which means that no emergency control action is necessary if the index is higher than this threshold.

\section{Conclusion}

A measurement-based power system instability early warning index was proposed. The verification results prove that the proposed index can indicate the potential instability of the system. The preliminary results have shown that the instability threshold can be used to early warn the instability caused by cascading outages. Future 


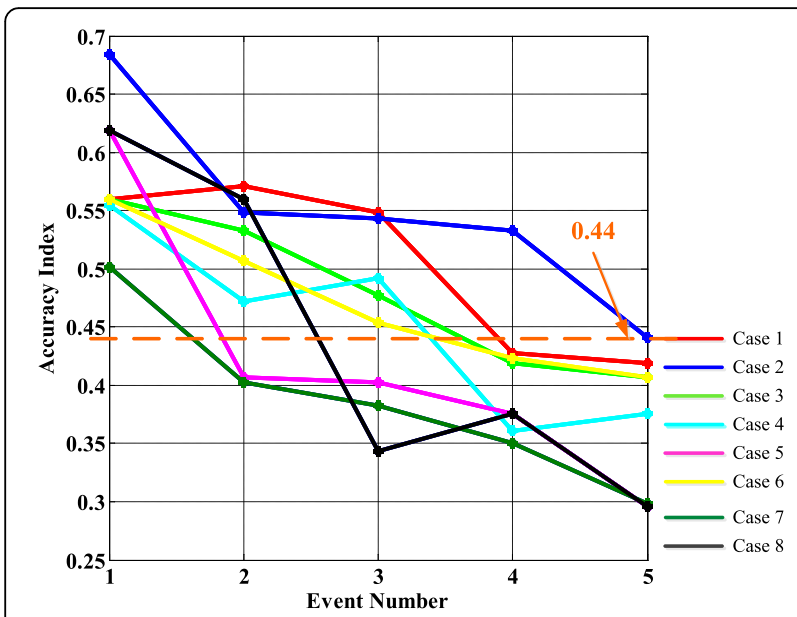

Fig. 6 Accuracy variation trends following the sequences of disturbances in 8 cases

work will focus on how to prove the effectiveness of the measurement-based instability early warning approach theoretically and more studies to explore the possibility of the application in the real power system.

\section{About the authors}

Feifei Bai (SM'13-M'16) received the B.S. and Ph.D. degree from the southwest Jiaotong Univeristy, Chengdu, China, in 2010 and 2016 respectively. She was a joint-Ph.D. student at the University of Tennessee, Knoxville, USA, from Sep. 2012 to Dec. 2014.

Her main research interests include small signal stability analysis and widearea damping control.

Yong Liu received the B.S. and M.S. degree in electrical engineering from Shandong University, Jinan, China, in 2007 and 2010 repectively, and the Ph.D. degree in electrical engineering from the University of Tennessee, Knoxville, TN, USA, in 2013.

He is currently a research assisstant professor with the DOE/NSF- cofunded Engineering Research Center CURENT, at the University of Tennessee, Knoxville, TN, USA. His research interests include power system wide-area measurement, large scale power system simulation, renewable energy integration and power system dynamic analysis.

Yilu Liu (S'88-M'89-SM'99-F'04) received her M.S. and Ph.D. degrees from the Ohio State University, Columbus, in 1986 and 1989. She received the B.S. degree from Xi'an Jiaotong University, China. She is currently the Governor's Chair at the University of Tennessee, Knoxville and Oak Ridge National Laboratory (ORNL). She is also the deputy Director of the DOE/NSF-cofunded engineering research center CURENT. Prior to joining UTK/ORNL, she was a Professor at Virginia Tech. She led the effort to create the North American power grid Frequency Monitoring Network (FNET) at Virginia Tech, which is now operated at UTK and ORNL as GridEye.

Her current research interests include power system wide-area monitoring and control, large interconnection-level dynamic simulations, electromagnetic transient analysis, and power transformer modeling and diagnosis.

Kai Sun (M'06-SM'13) received the B.S. degree inautomation in1999 and the Ph.D. degree in control science and engineering in 2004 both from Tsinghua University, Beijing, China. He was a postdoctoral research associate at Arizona State University, Tempe, from 2005 to 2007, and was a project manager in grid operations and planning areas at EPRI, PaloAlto, CA from 2007 to 2012. He iscurrently an assistant professor at the Department of EECS, University of Tennesseein Knoxville. His research interests include power system dynamics, stability and control and complex systems.

Navin B. Bhatt (F'09) received a BSEE degree from India, and MSEE and PhD degrees in electric power engineering from the West Virginia University, Morgantown, WV, USA. He works at EPRI since July 2010, where his activities focus on R\&D in the smart transmission grid, and transmission operations \& planning areas. Before joining EPRI, Dr. Bhatt worked at AEP for 33 years, where he conducted, managed and directed activities related to advanced analytical studies; managed AEP's transmission R\&D program; participated in the development of NERC standards; and participated in North American Synchrophasor Initiative (NASPI) activities.

Dr. Bhatt is a licensed Professional Engineer in Ohio. He has authored/co-authored over 50 technical papers. Dr. Bhatt was a member of the NERC technical team that investigated the August 14, 2003 blackout on behalf of the US and Canadian governments. He was a co-author of an IEEE working group paper that received in 2009 an award as an Outstanding Technical Paper. Dr. Bhatt has chaired 3 NERC teams and a NASPI task team.

Alberto Del Rosso (M'07) received his PhD degree from the Instituto de Energía Eléctrica, in Argentina, andElectromechanical Engineer diploma from the Universidad Tecnológica Nacional (UTN), Mendoza-Argentina. He was visiting researcher at the Department of Electrical \& Computer Engineer of the University of Waterloo in Ontario Canada.

Dr. Del Rosso is currently with EPRI, Knoxville, TN. He leads the EPRI's Transmission Modernization Demonstration (TMD) Initiative, and has also managed a variety of $R \& D$ and engineering projects related with transmission planning, smart grid technologies, transmission system operation optimization, dynamic security assessment, reactive power planning, wind generation integration, and vulnerability of nuclear power plants, among others. Formerly he worked for many years as power system consultant. He chairs and actively participates in several task forces and working groups at the CIGRE, IEEE and NERC

Evangelos Farantatos (S'06, M '13) was born in Athens, Greece in 1983. He received the Diploma in Electrical and Computer Engineering from the National Technical University of Athens, Greece, in 2006 and the M.S. in E.C.E. and Ph.D. degrees from the Georgia Institute of Technology in 2009 and 2012 respectively.

He is currently a Sr. Project Engineer/Scientist at EPRI. In summer 2009, he was an intern in MISO. His research interests include power systems state estimation, protection, stability, operation, control, synchrophasor applications, renewables integration and smart grid technologies. Xiaoru Wang (M'02-SM'07) received her B.S. degree and M.S. degree from Chongqing University, China, in 1983 and in1988 respectively, and a Ph.D. degree from Southwest Jiaotong University, China, in 1998. Since 2002, she has been a Professor in the School of Electrical Engineering, Southwest Jiaotong University.

Her research interests lie in the areas of power systems protection and emergency control. Since 2009, she has extended her research to modern power system with a large scale renewable power penetration.

\section{Acknowledgment}

The authors gratefully acknowledge FNET/GridEye group and all the EPRI project team members.

This work was partial supported by Electric Power Research Institute and also made use of Engineering Research Center Shared Facilities supported by the DOE under NSF Award Number EEC1041877 and the CURENT Industry Partnership Program. DOE under NSF Award Number EEC1041877 and the CURENT Industry Partership Program.

\section{Author details}

${ }^{1}$ Southwest Jiaotong University, Chengdu, Sichuan 610031, China. ${ }^{2}$ University of Tennessee, Knoxville, TN 37996, USA. ${ }^{3}$ Oak Ridge National Laboratory, 1 Bethel Valley Rd, Oak Ridge, TN 37831, USA. ${ }^{4}$ Electrical Power Research Institute, Palo Alto, CA 94304, USA.

Received: 10 May 2016 Accepted: 10 May 2016

Published online: 20 June 2016

\section{References}

1. IEEE/CIGRE Joint Task Force on Stability Terms and Definitions. Definition and classification of power system stability[]]. IEEE Trans Power Syst. 2004;19(3):1387-401.

2. Blankenship GL, Fink LH. Statistical characterizations of power system stability and security. In: Proc. $2^{\text {nd }}$ Lawrence Symp. Berkeley, CA: Systems and Decision Sciences; 1978. p. 62-70.

3. Andersson C. Power system security assessment:application of learning algorithms. Sweden: Lund University, Licentiate Thesis; 2005.

4. Ewart DN. Whys and wherefores of power system blackouts. IEEE Spectrum. 1978;15:36-41. 
5. Kamwa I, Grondin R. PMU configuration for system dynamic performance measurement in large multi area power systems. IEEE Trans Power Syst. 2002;17:385-94.

6. Sun K, Luo X, Wong JM. Early warning of wide-area angular stability problems using synchrophasors. San Diego: IEEE PES Power and Energy Society General Meeting; 2012. p. 1-6.

7. Sun K, Lee S, Zhang P. An adaptive power system equivalent for real time estimation of stability margin using phase-plane trajectories. IEEE Trans Power Syst. 2011;26:915-23.

8. Sun $\mathrm{K}$ et al. An online dynamic security assessment scheme using phasor measurements and decision trees. IEEE Trans Power Syst. 2007;22:1935-43.

9. Ma J et al. Use multi-dimensional ellipsoid to monitor dynamic behavior of power systems based on PMU measurement. In: Proc. IEEE PES General Meeting. 2008.

10. Quintero J, Liu G, Venkatasubramanian V. An oscillation monitoring system for real-time detection of small signal instability in large electric power systems. In: Proc. IEEE PES General Meeting. 2007.

11. Messina AR et al. Interpretation and visualization of wide-area PMU measurements using Hilbert analysis. IEEE Trans Power Syst. 2006;21:1763-71

12. Liu Y, Sun K, Liu Y. Measurement-based power system dynamic model for response estimation. San Diego: IEEE PES General Meeting; 2012. p. 1-6.

13. Eriksson $\mathrm{R}$, So X, Der L. Wide-area measurement system-based subspace identification for obtaining linear models to centrally coordinate controllable devices. IEEE Trans Power Del. 2011;26(2):988-97.

14. Kamwa I, Gerin-lajoie L. State-space system identification-toward MIMO models for modal analysis and optimization of bulk power systems. IEEE Trans Power Syst. 2000;15(1):326-35.

15. Zhou N, Pierre J, Hauer JF. Initial results in power system identification from injected probing signals using a subspace method. IEEE Trans Power Syst. 2006;21(3):1296-302

16. Ghasemi H, Canizares C, Moshref A. Oscillatory stability limit prediction using stochastic subspace identification. IEEE Trans Power Syst. 2006;21 (2):736-45.

17. Sarmadi SAN, Venkatasubramanian V. Electromechanical mode estimation using recursive adaptive stochastic subspace identification. IEEE Trans Power Syst. 2014;29(1):349-58.

18. Chaudhuri NR, Domahidi A, Majumder R, Chaudhuri B, Korba P, Ray S, Uhlen K. Wide-area power oscillation damping control in Nordic equivalent system. IET Gener Transm Distrib. 2010;4(10):1139-50.

19. Bai F, Liu Y, Liu Y, et al. Measurement-based correlation approach for power system dynamic response estimation. IET Gener Transm Distrib. 2015;9(12):1474-84.

20. Yao W, Jiang L, Wen JY, Wu QH, Cheng SJ. Wide-area damping controller for power system interarea oscillations: a networked predictive control approach. IEEE Trans Control Syst Technol. 2015;23(1):27-36.

21. Bai F, Liu Y, Sun K, Bhatt N, Rosso AD, Farantatos E, Wang X. Input Signal Selection for Measurement-Based Power System ARX Dynamic Model Response Estimation. Chicago, IL, US: IEEE PES T\&D Conference; 2014.

22. Kundur P. Power System Stability and Control. New York, NY, USA: McGraw-Hill; 1994

23. Ljung L. System identification: theory for the user. 2 nd ed. New Jersey: PTR Prentice; 1999. p. 1-296.

24. Liu Y, Sun K, Liu Y. A Measurement-based Power System Model for Dynamic Response Estimation and Instability Warning. Electr Power Syst Res. 2015;124:1-9.

25. Sun K, Hur K, Zhang P. A New Unified Scheme for Controlled Power System Separation Using Synchronized Phasor Measurements. IEEE Trans Power Systems. 2011;26(3):1544-1554.

\section{Submit your manuscript to a SpringerOpen ${ }^{\circ}$ journal and benefit from:}

- Convenient online submission

- Rigorous peer review

Immediate publication on acceptance

- Open access: articles freely available online

- High visibility within the field

- Retaining the copyright to your article

Submit your next manuscript at $\gg$ springeropen.com 